\title{
Thermal Green Functions in Coordinate Space for Massless Particles of any Spin
}

\author{
H. Arthur Weldon \\ Department of Physics, West Virginia University, Morgantown WV 26506-6315
}

(February 2, 2000)

The thermal Wightman functions for free, massless particles of spin $0,1 / 2,1,3 / 2$, and 2 are computed directly in coordinate space by solving the appropriate differential equation and imposing the Kubo-Martin-Schwinger condition. The solutions are valid for real, imaginary, or complex time. The Wightman functions for spin 1 gauge bosons and for spin 2 gravitons are directly related to the fundamental functions for spin 0 . The Wightman functions for spin $3 / 2$ gravitinos is directly related to that for spin $1 / 2$ fermions. Calculations for spin $1,3 / 2$, and 2 are done in covariant gauges. In the deep space-like region the Wightman functions for bosons fall like $T / r$ whereas those for the fermions fall exponentially. In the deep time-like region all the Wightman functions fall exponentially.

11.10.Wx, 12.38.Mh

\section{INTRODUCTION}

In both zero-temperature and finite-temperature field theory it is customary to perform calculations in momentum space. There are exceptions to this pattern however. Lattice gauge theory computations are done in coordinate space. The short-distance operator product expansion is formulated in coordinate space. It is even possible to carry out ultraviolet regularization and renormalization directly in coordinate space [1].

The purpose of this paper is to deduce the free Wightman functions at finite temperature for various massless particles directly in coordinate space. Thermal averages are performed with respect to the equilibrium density operator

$$
\varrho=e^{-\beta H} / \operatorname{Tr}\left[e^{-\beta H}\right],
$$

where $H$ is the appropriate Hamiltonian and $\beta=1 / T$. Let $\phi^{A}(x)$ denote a quantum field of any spin. For spinzero fields the index $A$ only distinguishes species. For spin $1 / 2$ fields $A$ denotes a spinor index; for spin 1 , a vector index; for spin $3 / 2$ a spinor and vector index; for spin 2, a pair of vector indices. The Wightman functions at finite temperature are

$$
\begin{aligned}
& G_{>}^{A B}(x)=-i \operatorname{Tr}\left(\varrho \phi^{A}(x) \phi^{B}(0)\right) \\
& G_{<}^{A B}(x)=-i \operatorname{Tr}\left(\varrho \phi^{B}(0) \phi^{A}(x)\right)(-1)^{2 J} .
\end{aligned}
$$

Knowing the thermal Wightman function allows direct construction of the various thermal propagators in real or imaginary time 2 2. 4 . In particular the time-ordered Green function is

$$
G^{A B}(x)=\theta(t) G_{>}^{A B}(x)+\theta(-t) G_{<}^{A B}(x) .
$$

The canonical method for obtaining $G_{>}(x)$ for free fields would require the following steps: (1) Solve the free field equations and express the field operator in terms of plane-wave solutions weighted by creation and annihilation operators. (2) Impose the equal-time canonical commutation relations. (3) Express the Hamiltonian in terms of creation and annihilation operators. (4) Compute the trace in Fock space over the density operator. (5) Perform the integral over momentum states so as to obtain $G_{>}(x)$ in coordinate space. For higher spins this becomes tedious.

The first four steps can be avoided if the $T=0$ propagator is already known, because the spectral function $\rho^{A B}(K)$ gives directly the Wightman function in momentum space [2 [4]:

$$
G_{>}^{A B}(K)=\frac{-i \rho^{A B}(K)}{1 \pm e^{-\beta k_{0}}} .
$$

However it is still necessary to Fourier transform from momentum space to coordinate space and obtain $G_{>}(x)$. This was the procedure followed in [5], which computed the coordinate-space Wightman functions for massless vector bosons in various gauges (Feynman, general covariant, and Coulomb). However performing the Fourier transforms so as to ensure the correct analyticity properties in complex time is difficult. For gauge bosons the complete answer satisfying all the analyticity properties was only obtained in the Feynman gauge. It would be laborious to pursue the Fourier transform method for spin $1 / 2$ fermions, for gravitinos, or for gravitons.

It turns out to be simpler to deduce the thermal Wightman functions for free fields by working directly in coordinate space. Since $G_{>}(x)$ solves the free field equation all that is necessary is that the solution have the correct zero-temperature limit and satisfy the Kubo-MartinSchwinger periodicity relation [2, 4, 6] under $t \rightarrow t-i \beta$. It has not been generally recognized that the KMS relation is not only a necessary condition that thermal Wightman functions must satisfy but is also sufficient condition to determine them directly.

The paper is organized according to spin. Sec II deals with bosons. The thermal Wightman functions for spinless bosons is given in Eq. (2.10), for gauge bosons in Eq. (2.17), and for gravitons in Eq. (2.22) and (2.28). Sec III deals with fermions. The thermal Wightman functions for spin $1 / 2$ fermions is given by Eq. (3.3) and (3.8); for spin $3 / 2$ gravitinos in Eq. (3.12) and (3.14). In the covariant gauges considered here, the higher spin functions 
are all expressible in terms of the basic spin 0 and $1 / 2$ functions. Sec III discusses the asymptotic behavior.

Throughout the paper the fundamental functions depend only radial distance $r$ and time $t$ and it is convenient to use variables $u$ and $v$ defined as follows:

$$
u=r+t \quad v=r-t
$$

To avoid confusion with the metric $g_{\mu \nu}$ in curved space, the Minkowski metric will everywhere be denoted by $\eta_{\mu \nu}$.

\section{BOSONS OF SPIN $0,1,2$}

\section{A. Spinless Bosons}

For a spinless boson field $\phi(x)$ the basic thermal Wightman functions are

$$
\begin{aligned}
& D_{>}(x)=-i \operatorname{Tr}(\varrho \phi(x) \phi(0)) \\
& D_{<}(x)=-i \operatorname{Tr}(\varrho \phi(0) \phi(x)) .
\end{aligned}
$$

The emphasis of the subsequent development will be to avoid expressing the free field operator as a sum of plane waves weighted by creation and annihilation operators. It will be more direct to solve the Klein-Gordon equation $\square D_{>}(x)=\square D_{<}(x)=0$ subject to various conditions. One such constraint is the normalization condition provided by imposing the canonical value of the equal-time commutator $[\dot{\phi}, \phi]$. This requires

$$
\left.\frac{\partial}{\partial t}\left(D_{>}(x)-D_{<}(x)\right)\right|_{t=0}=-\delta^{3}(\vec{r}) .
$$

The zero-temperature solution to the homogeneous differential equation satisfying the above initial condition is

$$
\begin{aligned}
\left.D_{>}(x)\right|_{T=0} & =\frac{i}{4 \pi^{2}} \frac{1}{(t-i \epsilon)^{2}-r^{2}} \\
\left.D_{<}(x)\right|_{T=0} & =\frac{i}{4 \pi^{2}} \frac{1}{(t+i \epsilon)^{2}-r^{2}} .
\end{aligned}
$$

At zero temperature $D_{>}(x)$ is analytic throughout the lower-half of the complex $t$ plane and $D_{<}(x)$ is analytic throughout the upper-half of the complex $t$ plane. For later purposes it will be convenient to write these in terms of the variables $u$ and $v$ of Eq. (1.5):

$$
\begin{aligned}
& \left.D_{>}(x)\right|_{T=0}=\frac{-i}{8 \pi^{2} r}\left[\frac{1}{u-i \epsilon}+\frac{1}{v+i \epsilon}\right] \\
& \left.D_{<}(x)\right|_{T=0}=\frac{-i}{8 \pi^{2} r}\left[\frac{1}{u+i \epsilon}+\frac{1}{v-i \epsilon}\right]
\end{aligned}
$$

The finite-temperature Wightman functions have some standard properties [2 that are easily demonstrated by inserting a complete set of energy eigenstates between the operators in (2.1). First, $D_{>}(x)$ is an analytic function of complex time in the strip $-\beta \leq \operatorname{Im} t \leq 0$, which will be referred to as the lower strip. Similarly $D_{>}(x)$ is analytic in complex time in the strip $0 \leq \operatorname{Im} t \leq \beta$, referred to as the upper strip. The values of each Wightman functions on the boundaries of its region of analyticity are related by the Kubo-Martin-Schwinger (KMS) conditions [2. 4 .

$$
\begin{aligned}
& D_{>}(t-i \beta, r)=D_{<}(t, r) \\
& D_{<}(t+i \beta, r)=D_{>}(t, r)
\end{aligned}
$$

The special property of free fields that will be essential is that their commutator $[\phi(x), \phi(0)]$ is a c-number at all $x$. The difference of the two Wightman functions is the thermal average of this c-number and therefore the difference is independent of temperature:

$$
D_{>}(x)-D_{<}(x)=\left.D_{>}(x)\right|_{T=0}-\left.D_{<}(x)\right|_{T=0} .
$$

This implies that the Wightman functions have the structure

$$
\begin{gathered}
D_{>}(x)=\left.D_{>}(x)\right|_{T=0}+E(x) \\
D_{<}(x)=\left.D_{<}(x)\right|_{T=0}+E(x) .
\end{gathered}
$$

Since $D_{>}(x)$ is analytic in the lower strip and $D_{<}(x)$ analytic in the upper strip, the function $E(x)$ is actually analytic in the double-width strip $-\beta \leq \operatorname{Im} t \leq \beta$.

Because the fields satisfy the massless Klein-Gordon equation, then $E(x)$ does too: $\square E(x)=0$. The most general spherically symmetric solution has the form

$$
E(x)=\frac{1}{r}\left(F_{1}(u)+F_{2}(v)\right) .
$$

From the definitions in Eq. 2.1), time translation invariance implies that $D_{>}(t, r)=D_{<}(-t, r)$. Consequently $E(x)$ must be an even function of time. This makes the two functions are the same: $F_{1}=F_{2}$. Therefore the Wightman functions have the form

$$
\begin{aligned}
& D_{>}(x)=\frac{-i}{8 \pi^{2} r}\left[\frac{1}{u-i \epsilon}+F(u)+\frac{1}{v+i \epsilon}+F(v)\right] \\
& D_{<}(x)=\frac{-i}{8 \pi^{2} r}\left[\frac{1}{u+i \epsilon}+F(u)+\frac{1}{v-i \epsilon}+F(v)\right] .
\end{aligned}
$$

The KMS condition Eq. (2.4) requires that $F$ satisfy

$$
\frac{1}{u+i \epsilon}+F(u)=\frac{1}{u-i \beta-i \epsilon}+F(u-i \beta) .
$$

This fixes $F(u)$ to have an infinite number of simple poles:

$$
F(u)=\sum_{n=1}^{\infty}\left[\frac{1}{u-i(n \beta+\epsilon)}+\frac{1}{u+i(n \beta+\epsilon)}\right] .
$$

This is the complete answer and has all the correct properties. Since $\operatorname{Im} u=\operatorname{Im} t$, there are no singularites in the closed strip $-\beta \leq \operatorname{Im} t \leq \beta$ as expected. The nearest 
poles are outside this strip at $u= \pm i(\beta+\epsilon)$. It is relatively easy to sum this series and obtain a more useful expression.

Complex time in the open strip: Often one is interested in complex time in the open strip

$$
-\beta<\operatorname{Im} t<\beta
$$

which of course includes real time. For the open strip, one can set $\epsilon \rightarrow 0$. Then the series sums to

$$
F(u)=-\frac{1}{u}+\pi T \operatorname{coth}(\pi T u) .
$$

This has no pole at $u=0$ but does have poles at the border $u= \pm i \beta$ because of the limit taken. Substituting this into Eq. (2.6) gives

$$
\begin{aligned}
D_{>}(t, r)= & \frac{1}{8 \pi r}(\delta(u)-\delta(v)) \\
& -\frac{i T}{8 \pi r}(\operatorname{coth}(\pi T u)+\operatorname{coth}(\pi T v)) \\
D_{<}(t, r)= & \frac{1}{8 \pi r}(-\delta(u)+\delta(v)) \\
& -\frac{i T}{8 \pi r}(\operatorname{coth}(\pi T u)+\operatorname{coth}(\pi T v))
\end{aligned}
$$

Note that it is only possible for $t$ and $t-i \beta$ to both be in the open strip defined in Eq. (2.9) if both are complex. But if both are complex then the Dirac delta functions have no support. Consequently the KMS condition is satisfied trivially here. It will be satisfied in a nontrivial fashion below in Eq. (2.14).

Imaginary time: The imaginary time formalism uses $t=-i \tau$ where $\tau$ is real. Then $u=r-i \tau$ and $v=r+i \tau$. The two Wightman functions are equal, $D_{>}(-i \tau, r)=$ $D_{<}(-i \tau, r)$ and given by

$$
D(-i \tau, r)=\frac{-i T}{4 \pi r} \frac{\sinh (2 \pi T r)}{\cosh (2 \pi T r)-\cos (2 \pi T \tau)}
$$

Naturally this is periodic under $\tau \rightarrow \tau \pm \beta$.

Arbitrary complex time: To treat all the poles of $F(u)$ in Eq. (2.8) correctly one cannot use the limit $\epsilon \rightarrow 0$. For non-zero $\epsilon$ the series can be summed in terms of the standard function $\psi(z)=d \ln [\Gamma(z)] / d z$ to obtain

$$
F(u)=i T \psi[1+T(\epsilon-i u)]-i T \psi[1+T(\epsilon+i u)] .
$$

This is analytic in the closed strip $-\beta \leq \operatorname{Im} t \leq \beta$ as expected.

Concise notation: For later purposes it is convenient to express $F(u)$ as a derivative. Therefore define a new function

$$
f(u)=\Gamma[1+T(\epsilon-i u)] \Gamma[1+T(\epsilon+i u)] .
$$

Then $F(u)=-\partial \ln [f(u)] / \partial r$ so that

$$
\frac{1}{r} F(u)=-\frac{1}{r} \frac{\partial}{\partial r} \ln [f(u)]=\frac{1}{2} \square \ln [f(u)] .
$$

Comparison with Eq. (2.6) shows that the thermal Wightman functions can be concisely expressed as

$$
\begin{aligned}
& D_{>}(x)=\square d_{>}(x) \\
& D_{<}(x)=\square d_{<}(x) .
\end{aligned}
$$

The lower case functions $d(x)$ are given by

$$
\begin{aligned}
& d_{>}(x)=-\frac{i}{16 \pi^{2}} \ln \left[\frac{f(u) f(v)}{(u-i \epsilon)(v+i \epsilon)}\right] \\
& d_{<}(x)=-\frac{i}{16 \pi^{2}} \ln \left[\frac{f(u) f(v)}{(u+i \epsilon)(v-i \epsilon)}\right]
\end{aligned}
$$

Note that the difference, $d_{>}(x)-d_{<}(x)$, is independent of temperature.

Special case: For many purposes, such as real time, it is adequate to use the open strip given in Eq. (2.9), which results from $\epsilon \rightarrow 0$. In this limit

$$
\lim _{\epsilon \rightarrow 0} f(u)=\frac{\pi T u}{\sinh (\pi T u)} .
$$

This makes Eq. (2.14) rather simple. The $\pm i \epsilon$ remaining in Eq. (2.14) produce the correct light-cone singularities $\delta(u)$ and $\delta(v)$ displayed earlier. In two important cases these Dirac delta functions have no support: either $t$ is complex or $t$ is real but not on the light-cone. In either case one can omit the $\pm i \epsilon$ in Eq. (2.14) in which case the two functions are equal: $d_{>}(x)=d_{<}(x) \equiv d(x)$ where

$$
d(x)=\frac{i}{16 \pi^{2}} \ln [\sinh (\pi T u) \sinh (\pi T v)] .
$$

In this regime the two thermal Wightman functions are equal: $D_{>}(x)=D_{<}(x)=\square d(x)$.

\section{B. Spin 1 Gauge Bosons}

The same methods can be used to obtain the thermal Wightman functions for massless gauge bosons directly in coordinate space. In a general covariant gauge the Lagrangian density is

$$
\mathcal{L}=-\frac{1}{4} F_{\mu \nu} F^{\mu \nu}-\frac{1}{2 \xi}\left(\partial_{\mu} A^{\mu}\right)^{2},
$$

where $F_{\mu \nu}=\partial_{\mu} A_{\nu}-\partial_{\nu} A_{\mu}$ and $\xi$ is an arbitrary gauge parameter. The thermal Wightman functions are

$$
\begin{aligned}
& D_{>}^{\mu \nu}(x)=-i \operatorname{Tr}\left(\varrho A^{\mu}(x) A^{\nu}(0)\right) \\
& D_{<}^{\mu \nu}(x)=-i \operatorname{Tr}\left(\varrho A^{\nu}(0) A^{\mu}(x)\right) .
\end{aligned}
$$

One can deduce these from first principles using the same arguments as employed in Sec IIA. The subsequent discussion will show that

$$
\begin{aligned}
& D_{>}^{\mu \nu}(x)=\left(-\eta^{\mu \nu} \square+(1-\xi) \partial^{\mu} \partial^{\nu}\right) d_{>}(x) \\
& D_{<}^{\mu \nu}(x)=\left(-\eta^{\mu \nu} \square+(1-\xi) \partial^{\mu} \partial^{\nu}\right) d_{<}(x),
\end{aligned}
$$


where $d_{>}(x)$ and $d_{<}(x)$ are the functions functions already given in Eq. (2.14).

(1) The first check of these Wightman functions is that they have the correct analyticity in complex time and satisfy the correct KMS condition

$$
D_{>}^{\mu \nu}(t-i \beta, \vec{r})=D_{<}^{\mu \nu}(t, \vec{r}) .
$$

(2) The next check is that the Wightman functions must satisfy the correct homogeneous differential equation. The variation of the Lagrangian gives

$$
\frac{\partial \mathcal{L}}{\partial\left(\partial^{\rho} A^{\kappa}\right)}=P_{\rho \kappa \mu} A^{\mu}
$$

where the tensor $P$ is linear in the first derivative:

$$
P_{\rho \kappa \mu}=\eta_{\rho \mu} \partial_{\kappa}-\eta_{\kappa \mu} \partial_{\rho}-\frac{1}{\xi} \eta_{\rho \kappa} \partial_{\mu} .
$$

The equation of motion for the field is

$$
0=\partial^{\rho} P_{\rho \kappa \mu} A^{\mu}=\left[-\eta_{\kappa \mu} \square+\left(1-\frac{1}{\xi}\right) \partial_{\kappa} \partial_{\mu}\right] A^{\mu} .
$$

Applying this differential operator to the Wightman function in Eq. (2.17) gives

$$
\left(-\eta_{\lambda \mu} \square+\left(1-\frac{1}{\xi}\right) \partial_{\lambda} \partial_{\mu}\right) D_{>}^{\mu \nu}(x)=-\delta_{\lambda}^{\nu} \square \square d_{>}(x) .
$$

From Sec IIA, $\square \square d_{>}(x)=\square D_{>}(x)=0$ and so the equation of motion is satisfied.

(3) The third check is that the field operators in the Wightman functions satisfy the correct canonical commutation relations. The canonical momentum conjugate to $A^{\kappa}$ is

$$
\Pi_{\kappa}=P_{0 \kappa \mu} A^{\mu} \text {. }
$$

The equal-time canonical commutation relations are

$$
-i\left[\Pi_{\kappa}(x), A^{\nu}(0)\right]_{t=0}=-\delta_{\kappa}^{\nu} \delta^{3}(\vec{r}) .
$$

This requires that the Wightman functions to satisfy

$$
\left[P_{0 \kappa \mu}\left(D_{>}^{\mu \nu}(x)-D_{<}^{\mu \nu}(x)\right)\right]_{t=0}=-\delta_{\kappa}^{\nu} \delta^{3}(\vec{r}) .
$$

To check this, use Eq. (2.17) and (2.19) to obtain

$$
P_{\rho \kappa \mu} D_{>}^{\mu \nu}(x)=\left(\delta_{\kappa}^{\nu} \partial_{\rho}-\delta_{\rho}^{\nu} \partial_{\kappa}+\eta_{\rho \kappa} \partial^{\nu}\right) D_{>}(x),
$$

where $D_{>}(x)$ is the thermal Wightman function for scalars. For the appropriate difference of Wightman functions one needs

$$
\begin{aligned}
& P_{0 \beta \mu}\left(D_{>}^{\mu \nu}(x)-D_{<}^{\mu \nu}(x)\right) \\
& \quad=\left(\delta_{\kappa}^{\nu} \partial_{0}-\delta_{0}^{\nu} \partial_{\kappa}+\eta_{0 \kappa} \partial^{\nu}\right)\left(D_{>}(x)-D_{<}(x)\right) .
\end{aligned}
$$

The right hand side contains both time derivatives and space derivatives. At $t=0$ the spatial derivatives vanish because $D_{>}(0, \vec{r})=D_{<}(0, \vec{r})$. The above result simplifies to

$$
\left[P_{0 \beta \mu}\left(D_{>}^{\mu \nu}(x)-D_{<}^{\mu \nu}(x)\right)\right]_{t=0}=\delta_{\kappa}^{\nu}\left[\left(\dot{D}_{>}(x)-\dot{D}_{<}(x)\right]_{t=0} .\right.
$$

Eq. (2.2) determines that the value of the right hand side is $-\delta^{\nu} \delta^{3}(\vec{r})$, as required. This completes the proof that Eq. 2.17) is correct.

\section{Spin 2 Gravitons}

Standard quantum gravity is based on the EinsteinHilbert Lagrangian with a gauge-fixing term

$$
\mathcal{L}=\frac{2}{\kappa^{2}} \sqrt{-g} R+\mathcal{L}_{\text {g.f. }}
$$

in which $R$ is the scalar curvature, $g=\operatorname{det}\left(g_{\mu \nu}\right)$, and $\kappa^{2}=32 \pi G$ with $G$ Newton's constant. A conventional gauge fixing term is $[7,8$

$$
\mathcal{L}_{\text {g.f. }}=\frac{1}{k^{2}} \eta_{\mu \nu}\left(\partial_{\alpha} \sqrt{-g} g^{\alpha \mu}\right)\left(\partial_{\beta} \sqrt{-g} g^{\beta \nu}\right) .
$$

This corresponds to the Feynman gauge in Yang-Mills theories. More general covariant gauges will be discussed later. With $\eta^{\mu \nu}$ the Minkowski metric, the graviton field $h^{\mu \nu}$ contains all quantum fluctuations:

$$
\sqrt{-g} g^{\mu \nu}=\eta^{\mu \nu}+\kappa h^{\mu \nu}
$$

Keeping only terms that are quadratic in $h$ produces the free Lagrangian density

$$
\begin{aligned}
\mathcal{L}_{0}= & \frac{1}{2}\left(\partial_{\rho} h_{\alpha \beta}\right)\left(\partial^{\rho} h^{\alpha \beta}\right)-\frac{1}{4}\left(\partial_{\rho} h_{\alpha}{ }^{\alpha}\right)\left(\partial^{\rho} h_{\beta}{ }^{\beta}\right) \\
& -\left(\partial_{\rho} h_{\alpha \beta}\right)\left(\partial^{\alpha} h^{\rho \beta}\right)+\left(\partial_{\rho} h^{\rho \alpha}\right)\left(\partial^{\beta} h_{\beta \alpha}\right) .
\end{aligned}
$$

The thermal Wightman functions to be computed are

$$
\begin{aligned}
& D_{>}^{\mu \nu \alpha \beta}(x)=-i \operatorname{Tr}\left(\varrho h^{\mu \nu}(x) h^{\alpha \beta}(0)\right) \\
& D_{<}^{\mu \nu \alpha \beta}(x)=-i \operatorname{Tr}\left(\varrho h^{\alpha \beta}(0) h^{\mu \nu}(x)\right) .
\end{aligned}
$$

Subsequent argument will show that these are given by

$$
\begin{aligned}
& D_{>}^{\mu \nu \alpha \beta}(x)=\left(-\eta^{\mu \nu} \eta^{\alpha \beta}+\eta^{\mu \alpha} \eta^{\nu \beta}+\eta^{\mu \beta} \eta^{\nu \beta}\right) D_{>}(x) \\
& D_{<}^{\mu \nu \alpha \beta}(x)=\left(-\eta^{\mu \nu} \eta^{\alpha \beta}+\eta^{\mu \alpha} \eta^{\nu \beta}+\eta^{\mu \beta} \eta^{\nu \beta}\right) D_{<}(x) .
\end{aligned}
$$

That the results are expressed in terms of the scalar Wightman functions $D_{>}(x)$ rather than the potential functions $d_{>}(x)$ is a peculiarity of this Feynman-like gauge. In more general covariant gauges the Wightman functions for gravitons depends on the potentials $d_{>}(x)$.

(1) Because of the properties of the scalar Wightman functions, the graviton Wightman functions in Eq. 2.22) satisfy

$$
D_{>}^{\mu \nu \alpha \beta}(t-i \beta, \vec{r})=D_{>}^{\mu \nu \alpha \beta}(t, \vec{r}),
$$

and are analytic in the appropriate regions.

(2) To obtain the equation for the graviton field one needs the partial derivatives

$$
\frac{\partial \mathcal{L}}{\partial\left(\partial^{\rho} h^{\kappa \lambda}\right)}=P_{\rho \kappa \lambda \mu \nu} h^{\mu \nu}
$$

where the tensor $P$ is linear in the derivative operator 


$$
\begin{aligned}
P_{\rho \kappa \lambda \mu \nu} & =\left(\Lambda_{\kappa \lambda \mu \nu}-\frac{1}{2} \eta_{\kappa \lambda} \eta_{\mu \nu}\right) \partial_{\rho} \\
& -\Lambda_{\rho \lambda \mu \nu} \partial_{\kappa}-\Lambda_{\kappa \rho \mu \nu} \partial_{\lambda} \\
& +\Lambda_{\kappa \lambda \rho \nu} \partial_{\mu}+\Lambda_{\kappa \lambda \mu \rho} \partial_{\nu}
\end{aligned}
$$

and $\Lambda$ is given by

$$
\Lambda_{\kappa \lambda \mu \nu}=\frac{1}{2}\left(\eta_{\kappa \mu} \eta_{\lambda \nu}+\eta_{\kappa \nu} \eta_{\lambda \mu}\right) .
$$

The differential equation for the graviton field operator is $\partial^{\rho} P_{\rho \kappa \lambda \mu \nu} h^{\mu \nu}(x)=0$, or more concisely

$$
\left(-\eta_{\kappa \lambda} \eta_{\mu \nu}+\eta_{\kappa \mu} \eta_{\lambda \nu}+\eta_{\kappa \nu} \eta_{\lambda \mu}\right) \square h^{\mu \nu}(x)=0 .
$$

The graviton Wightman functions Eq. (2.22) automatically satisfy $\square D_{>}^{\mu \nu \alpha \beta}(x)=0$ since the scalar functions satisfy $\square D_{>}(x)=0$.

(3) The third check of Eq. (2.22) is that the graviton operators satisfy the correct canonical commutation relations. The canonical momentum conjugate to $h^{\kappa \lambda}$ is

$$
\Pi_{\kappa \lambda}=P_{0 \kappa \lambda \mu \nu} h^{\mu \nu} .
$$

The canonical equal-time commutation relations are

$$
-i\left[\Pi_{\kappa \lambda}(x), h^{\alpha \beta}(0)\right]_{t=0}=-\left(\delta_{\kappa}^{\alpha} \delta_{\lambda}^{\beta}+\delta_{\kappa}^{\beta} \delta_{\lambda}^{\alpha}\right) \delta^{3}(\vec{r}) .
$$

Therefore the Wightman functions should satisfy

$$
\begin{aligned}
& {\left[P_{0 \kappa \lambda \mu \nu}\left(D_{>}^{\mu \nu \alpha \beta}(x)-D_{<}^{\mu \nu \alpha \beta}(x)\right)\right]_{t=0}} \\
& =-\left(\delta_{\kappa}^{\alpha} \delta_{\lambda}^{\beta}+\delta_{\kappa}^{\beta} \delta_{\lambda}^{\alpha}\right) \delta^{3}(\vec{r}) .
\end{aligned}
$$

To check that the Wightman function Eq. (2.22) satisfies this, first apply the differential operator in Eq. (2.24):

$$
\begin{aligned}
P_{\rho \kappa \lambda \mu \nu}\left(D_{>}^{\mu \nu \alpha \beta}(x)-D_{>}^{\mu \nu \alpha \beta}(x)\right) \\
=\left(\delta_{\kappa}^{\alpha} \delta_{\lambda}^{\beta}+\delta_{\kappa}^{\beta} \delta_{\lambda}^{\alpha}\right) \partial_{\rho}\left(D_{>}(x)-D_{<}(x)\right) \\
-\left(\delta_{\rho}^{\alpha} \delta_{\lambda}^{\beta}+\delta_{\rho}^{\beta} \delta_{\lambda}^{\alpha}\right) \partial_{\kappa}\left(D_{>}(x)-D_{<}(x)\right) \\
-\left(\delta_{\kappa}^{\alpha} \delta_{\rho}^{\beta}+\delta_{\kappa}^{\beta} \delta_{\rho}^{\alpha}\right) \partial_{\lambda}\left(D_{>}(x)-D_{<}(x)\right) \\
+\left(\eta_{\rho \kappa} \delta_{\lambda}^{\beta}+\eta_{\rho \lambda} \delta_{\kappa}^{\beta}\right) \partial^{\alpha}\left(D_{>}(x)-D_{<}(x)\right) \\
+\left(\eta_{\rho \kappa} \delta_{\lambda}^{\alpha}+\eta_{\rho \lambda} \delta_{\kappa}^{\alpha}\right) \partial^{\beta}\left(D_{>}(x)-D_{<}(x)\right) .
\end{aligned}
$$

Now set the index $\rho=0$ and the time $t=0$. The first line obviously coincides with Eq. (2.27) and it is straightforward to check that the remaining four lines will always vanish. For example, if all of the free indices $\kappa, \lambda, \mu, \nu$ are spatial, the last four lines vanish. This, because the spatial derivatives on the right hand side are zero since $D_{>}(0, \vec{r})=D_{<}(0, \vec{r})$. If three of the indices $\kappa, \lambda, \mu, \nu$ are spatial and one is 0 , there will be one non-vanishing time derivatives among the last four lines but it will be multiplied by a tensor that vanishes. For the remaining cases it is easy to enumerate the possible values of the free indices $\kappa, \lambda, \mu, \nu$ and verify that the canonical commutation relation Eq. (2.27) is fully satisfied.
General Covariant Gauge: The results for the Wightman function given in Eq. (2.22) are for a particular gauge analogous to the Feynman gauge. A more general gauge-fixing term is

$$
\mathcal{L}_{\text {g.f. }}=\frac{1}{\xi k^{2}} \eta_{\mu \nu}\left(\partial_{\alpha} \sqrt{-g} g^{\alpha \mu}\right)\left(\partial_{\beta} \sqrt{-g} g^{\beta \nu}\right) .
$$

where $\xi$ is arbitrary. The differential equation for the graviton field $h^{\mu \nu}(x)$ is then

$$
\begin{aligned}
& \left(-\eta_{\kappa \lambda} \eta_{\mu \nu}+\eta_{\kappa \mu} \eta_{\lambda \nu}+\eta_{\kappa \nu} \eta_{\lambda \mu}\right) \square h^{\mu \nu} \\
& =\left(1-\frac{1}{\xi}\right)\left(\eta_{\kappa \mu} \partial_{\lambda} \partial_{\nu}+\eta_{\kappa \nu} \partial_{\lambda} \partial_{\mu}+\eta_{\lambda \mu} \partial_{\kappa} \partial_{\nu}+\eta_{\lambda \nu} \partial_{\kappa} \partial_{\mu}\right) h^{\mu \nu} .
\end{aligned}
$$

Following the same procedures as above one can show that the thermal Wightman function is

$$
\begin{gathered}
D_{>}^{\mu \nu \alpha \beta}(x)=\left(-\frac{1}{\xi} \eta^{\mu \nu} \eta^{\alpha \beta}+\eta^{\mu \alpha} \eta^{\nu \beta}+\eta^{\mu \beta} \eta^{\nu \beta}\right) \square d_{>}(x) \\
+\left(1-\frac{1}{\xi}\right)\left\{-2\left(\eta^{\mu \nu} \partial^{\alpha} \partial^{\beta}+\eta^{\alpha \beta} \partial^{\mu} \partial^{\nu}\right) d_{>}(x)\right. \\
\left.+\left(\eta^{\mu \alpha} \partial^{\nu} \partial^{\beta}+\eta^{\mu \beta} \partial^{\nu} \partial^{\alpha}+\eta^{\nu \alpha} \partial^{\mu} \partial^{\beta}+\eta^{\nu \beta} \partial^{\mu} \partial^{\alpha}\right) d_{>}(x)\right\} .
\end{gathered}
$$

Note that the general Wightman function depends on all partial derivatives of $d_{>}(x)$, whereas Eq. (2.22), in the Feynman-like gauge, depends only on $D_{>}(x)=\square d_{>}(x)$.

\section{FERMIONS}

The methods employed in the previous section carry over to fermions except that the KMS condition contains an additional minus sign. This change results in a completely different asymptotic behavior in the deep space-like region.

\section{A. Spin 1/2 Fermions}

The thermal Wightman functions for spin- $1 / 2$ fields are given by

$$
\begin{aligned}
& S_{>\alpha \beta}(x)=-i \operatorname{Tr}\left(\varrho \psi_{\alpha}(x) \bar{\psi}_{\beta}(0)\right) \\
& S_{<\alpha \beta}(x)=i \operatorname{Tr}\left(\varrho \bar{\psi}_{\beta}(0) \psi_{\alpha}(x)\right) .
\end{aligned}
$$

The ordering of the spinor indices is important, but the indices will be suppressed in the following. The relative sign difference between the two functions is conventional.

At zero temperature the Wightman functions are

$$
\begin{aligned}
\left.S_{>}(x)\right|_{T=0} & =i \gamma \cdot \partial \frac{i}{4 \pi^{2}} \frac{1}{(t-i \epsilon)^{2}-r^{2}} \\
\left.S_{<}(x)\right|_{T=0} & =i \gamma \cdot \partial \frac{i}{4 \pi^{2}} \frac{1}{(t+i \epsilon)^{2}-r^{2}} .
\end{aligned}
$$

The former is analytic in the entire lower-half of the complex $t$ plane and the upper is analytic in the entire upperhalf of that plane. 
At nonzero temperature the regions of analyticity are reduced. In the complex time plane, $S_{>}(x)$ is analytic in the lower strip $-\beta \leq \operatorname{Im} t \leq 0$ and $S_{<}(x)$ is analytic in the upper strip $0 \leq \operatorname{Im} t \leq \beta$. On the boundaries of these regions the values are related by the KMS conditions [6]:

$$
\begin{aligned}
& S_{>}(t-i \beta, \vec{r})=-S_{<}(t, \vec{r}) \\
& S_{<}(t+i \beta, \vec{r})=-S_{>}(t, \vec{r}) .
\end{aligned}
$$

The difference between the Wightman functions is the anticommutator of the fields. For free fields this anticommutator is a c-number:

$$
S_{>\alpha \beta}(x)-S_{<\alpha \beta}(x)=-i\left\{\psi_{\alpha}(x), \bar{\psi}_{\beta}(0)\right\}
$$

and thus the right hand side is independent of temperature. This implies

$$
S_{>}(x)-S_{<}(x)=\left.S_{>}(x)\right|_{T=0}-\left.S_{<}(x)\right|_{T=0} .
$$

so that the Wightman functions have the structure

$$
\begin{aligned}
& S_{>}(x)=\left.S_{>}(x)\right|_{T=0}+E(x) \\
& S_{<}(x)=\left.S_{<}(x)\right|_{T=0}+E(x) .
\end{aligned}
$$

The free-field equation $-i \gamma \cdot \partial \psi=0$ implies that $E(x)$ must satisfy $-i \gamma \cdot \partial E=0$. To solve this, let the unknown function $E=i \gamma \cdot \partial F(x)$ where $\square F(x)=0$. The general spherically symmetric solution is

$$
E(x)=i \gamma \cdot \partial \frac{1}{r}\left(G_{1}(u)+G_{2}(v)\right) .
$$

From their definitions, the Wightman functions satisfy $\left[S_{>}(x)\right]^{\dagger}=\gamma_{0} S_{>}\left(-x^{*}\right) \gamma_{0}$ and this makes the two functions the same: $G_{1}=G_{2}$. Combining this with the previous zero-temperature results allows the full thermal Wightman function to be expressed as

$$
\begin{aligned}
& S_{>}(x)=(i \gamma \cdot \partial) \sigma_{>}(x) \\
& S_{<}(x)=(i \gamma \cdot \partial) \sigma_{<}(x),
\end{aligned}
$$

in which the new functions have the form

$$
\begin{aligned}
& \sigma_{>}(x)=\frac{-i}{8 \pi^{2} r}\left[\frac{1}{u-i \epsilon}+G(u)+\frac{1}{v+i \epsilon}+G(v)\right] \\
& \sigma_{>}(x)=\frac{-i}{8 \pi^{2} r}\left[\frac{1}{u+i \epsilon}+G(u)+\frac{1}{v-i \epsilon}+G(v)\right],
\end{aligned}
$$

All the temperature dependence is contained in the single unknown function $G$. It will be determined by the fermionic KMS condition Eq. (3.1), which requires that $\sigma_{>}(t-i \beta, r)=-\sigma_{<}(t, r)$. For the function $G$ this requires that

$$
\frac{1}{u-i(\beta+\epsilon)}+G(u-i \beta)=-\left(\frac{1}{u+i \epsilon}+G(u)\right) .
$$

The solution for $G$ is

$$
G(u)=\sum_{n=1}^{\infty}(-1)^{n}\left[\frac{1}{u-i(n \beta+\epsilon)}+\frac{1}{u+i(n \beta+\epsilon)}\right] .
$$

This is analytic in the closed strip $-\beta \leq \operatorname{Im} t \leq \beta$. The nearest poles are just above this strip at $r+t=i(\beta+\epsilon)$ and just below the strip at $r+t=-i(\beta+\epsilon)$. The alternating signs will produce more rapid convergence than in the bosonic case.

Complex time in the open strip: For many purposes one is interested in either in real time or in complex time in the open strip

$$
-\beta<\operatorname{Im} t<\beta
$$

For $t$ in this open region one can set $\epsilon \rightarrow 0$ in Eq. (3.5) which allows the sum to be easily performed:

$$
G(u)=-\frac{1}{u}+\frac{\pi T}{\sinh (\pi T u)} .
$$

There is, of course, no pole at $u=0$. There are poles at $u= \pm \operatorname{in} \beta$ because of the limt $\epsilon \rightarrow 0$. The results for $\sigma_{>}$ and $\sigma_{<}$are

$$
\begin{aligned}
\sigma_{>}(x) & =\frac{1}{8 \pi r}(\delta(u)-\delta(v)) \\
& -\frac{i T}{8 \pi r}\left[\frac{1}{\sinh (\pi T u)}+\frac{1}{\sinh (\pi T v)}\right] \\
\sigma_{<}(x) & =\frac{1}{8 \pi r}(-\delta(u)+\delta(v)) \\
& -\frac{i T}{8 \pi r}\left[\frac{1}{\sinh (\pi T u)}+\frac{1}{\sinh (\pi T v)}\right] .
\end{aligned}
$$

It is worth noting that the KMS condition $\sigma_{>}(t-i \beta, r)=$ $-\sigma_{<}(t, \beta)$ is now satisfied in a trivial manner because of the restriction to the open strip in Eq. (3.6). Because of this, it is only possible for $t$ and $t-i \beta$ to lie in the open strip if both are complex and if both are complex then the Dirac delta functions have no support. In the full solution Eq. (3.5) the KMS condition is satisfied nontrivially.

Imaginary time: The imaginary time formalism uses $t=-i \tau$ where $\tau$ is real. As occurred for bosons, the two Wightman functions are equal, $\sigma_{>}(-i \tau, r)=\sigma_{<}(-i \tau, r)$ and given by

$$
\sigma(-i \tau, r)=\frac{-i T}{2 \pi r} \frac{\sinh (2 \pi T r) \cos (\pi T \tau)}{\cosh (2 \pi T r)-\cos (2 \pi T \tau)} .
$$

This is antiperiodic under $\tau \rightarrow \tau \pm \beta$.

Arbitrary complex time: Without approximation one can sum the series in Eq. (3.5) to obtain

$$
\begin{aligned}
G(u) & =i \frac{T}{2} \psi\left[\frac{1}{2}+\frac{T}{2}(\epsilon+i u)\right]-i \frac{T}{2} \psi\left[1+\frac{T}{2}(\epsilon+i u)\right] \\
& -i \frac{T}{2} \psi\left[\frac{1}{2}+\frac{T}{2}(\epsilon-i u)\right]+i \frac{T}{2} \psi\left[1+\frac{T}{2}(\epsilon-i u)\right] .
\end{aligned}
$$

Since $\psi(z)=d \ln [\Gamma(z)] / d z$ this is equivalent to 


$$
G(u)=\frac{\partial}{\partial r} \ln [g(u)] .
$$

in which the lower-case function $g$ is

$$
g(u)=\frac{\Gamma\left[\frac{1}{2}+\frac{T}{2}(\epsilon+i u)\right] \Gamma\left[\frac{1}{2}+\frac{T}{2}(\epsilon-i u)\right]}{\Gamma\left[1+\frac{T}{2}(\epsilon+i u)\right] \Gamma\left[1+\frac{T}{2}(\epsilon-i u)\right]}
$$

Putting this together in Eq. (3.4) gives the general result

$$
\begin{aligned}
& \sigma_{>}(x)=\square s_{>}(x) \\
& \sigma_{<}(x)=\square s_{<}(x) .
\end{aligned}
$$

in which the lower-case functions are

$$
\begin{aligned}
& s_{>}(x)=-\frac{i}{16 \pi^{2}} \ln \left[\frac{g(u) g(v)}{(u-i \epsilon)(v+i \epsilon)}\right] \\
& s_{<}(x)=-\frac{i}{16 \pi^{2}} \ln \left[\frac{g(u) g(v)}{(u+i \epsilon)(v-i \epsilon)}\right] .
\end{aligned}
$$

The next section will show will express the Wightman functions for gravitinos in terms of these functions.

\section{B. Spin 3/2 Gravitinos}

The massless Rarita-Schwinger field [9] plays an important role in supergravity as the spin $3 / 2$, supersymmetric partner of the graviton. In that context it is referred to as the gravitino. As occurred in the bosonic cases, the passage from the lower spin fermion $(1 / 2)$ to the higher spin fermion $(3 / 2)$ is easily accomplished. The free Lagrangian density for the field $\psi_{\mu}(x)$ is $[10$

$$
\mathcal{L}=-\epsilon^{\mu \nu \alpha \beta} \bar{\psi}_{\mu} \gamma_{\nu} \gamma_{5} \partial_{\alpha} \psi_{\beta}+\mathcal{L}_{g . f .} .
$$

The gauge fixing is necessary so as to break the invariance of the first term under transformations $\psi_{\beta} \rightarrow \psi_{\beta}+\partial_{\beta} \psi$, where $\psi$ is any spin- $1 / 2$ field. A convenient choice for gauge-fixing is

$$
\mathcal{L}_{g . f .}=\frac{i}{2} \bar{\psi}^{\nu} \gamma_{\nu}(\gamma \cdot \partial) \gamma_{\lambda} \psi^{\lambda}
$$

The free Lagrangian can be rewritten as

$$
\mathcal{L}=-\bar{\psi}^{\mu} L_{\mu \nu} \psi^{\nu},
$$

where the tensor $L_{\mu \nu}$ is linear in the first derivatives:

$$
L^{\mu \nu}=-i\left(\gamma^{\mu} \partial^{\nu}+\gamma^{\nu} \partial^{\mu}+\eta^{\mu \nu} \gamma \cdot \partial-\frac{1}{2} \gamma^{\mu}(\gamma \cdot \partial) \gamma^{\nu}\right) .
$$

Each Wightman functions is a $4 \times 4$ matrix in spinor space and a rank 2 tensor in the Lorentz indices:

$$
\begin{aligned}
& S_{>}^{\mu \nu}(x)=-i \operatorname{Tr}\left(\varrho \psi^{\mu}(x) \bar{\psi}^{\nu}(0)\right) \\
& S_{<}^{\mu \nu}(x)=i \operatorname{Tr}\left(\varrho \bar{\psi}^{\nu}(0) \psi^{\mu}(x)\right) .
\end{aligned}
$$

The spinor indices have been suppressed. The relative sign difference in the definitions coincides with the spin
$1 / 2$ convention. As in the bosonic case the most efficient way to proceed is to display the answer immediately and then perform checks. In this gauge the Wightman functions can be written in terms of the same matrixdifferential operator $L^{\mu \nu}$

$$
\begin{aligned}
& S_{>}^{\mu \nu}(x)=L^{\mu \nu} \sigma_{>}(x) \\
& S_{<}^{\mu \nu}(x)=L^{\mu \nu} \sigma_{<}(x),
\end{aligned}
$$

where $\sigma_{>}(x)$ and $\sigma_{<}(x)$ are the basic spin 1/2 functions given in Eq. (3.10). The subsequent discussion will confirm these results.

(1) The spin $1 / 2$ functions $\sigma_{>}(x)$ and $\sigma_{<}(x)$ guarantee that the gravitino Wightman functions in Eq. (3.14) satisfy the KMS conditions

$$
\begin{aligned}
& S_{>}^{\mu \nu}(t-i \beta, \vec{r})=-S_{<}^{\mu \nu}(t, \vec{r}) \\
& S_{<}^{\mu \nu}(t+i \beta, \vec{r})=-S_{>}^{\mu \nu}(t, \vec{r}),
\end{aligned}
$$

and have the correct analyticity properties.

(2) The equation of motion for the gravitino which follows directly from Eq. (3.11) is

$$
L_{\alpha \mu} \psi^{\mu}(x)=0 .
$$

Therefore the Wightman functions should satisfy

$$
L_{\alpha \mu} S_{>}^{\mu \nu}(x)=L_{\alpha \mu} S_{<}^{\mu \nu}(x)=0 .
$$

This is easily satisfied because of the identity

$$
L_{\alpha \mu} L^{\mu \nu}=-\delta_{\alpha}^{\mu} \square
$$

and the fact that $\square \sigma_{>}=0$ and $\square \sigma_{<}=0$.

(3) The third check of Eq. (3.14) is that the gravitino field operators must satisfy the canonical commutation relations at equal time. For this it is convenient to express the partial derivative of the Lagrangian as

$$
\frac{\partial \mathcal{L}}{\partial\left(\partial^{\rho} \psi^{\lambda}\right)}=\bar{\psi}^{\nu} \Gamma_{\nu \lambda \rho},
$$

where $\Gamma_{\nu \lambda \rho}$ is the matrix

$$
\Gamma_{\nu \lambda \rho} \equiv i\left(\gamma_{\nu} \eta_{\lambda \rho}+\gamma_{\lambda} \eta_{\nu \rho}-\eta_{\nu \lambda} \gamma_{\rho}-\frac{1}{2} \gamma_{\nu} \gamma_{\rho} \gamma_{\lambda}\right)
$$

Note that $L_{\nu \lambda}=-\Gamma_{\nu \lambda \rho} \partial^{\rho}$. The canonical momentum conjugate to $\psi^{\lambda}(x)$ is

$$
\Pi_{\lambda}(x)=\bar{\psi}^{\nu} \Gamma_{\nu \lambda 0} .
$$

These momenta obey the canonical equal-time anticommutation relations

$$
-i\left\{\psi^{\mu}(x), \Pi_{\lambda}(0)\right\}_{t=0}=-\delta_{\lambda}^{\mu} \delta^{3}(\vec{r}),
$$

so that the Wightman functions must satisfy

$$
\left[\left(S_{>}^{\mu \nu}(x)-S_{<}^{\mu \nu}(x)\right) \Gamma_{\nu \lambda 0}\right]_{t=0}=-\delta_{\lambda}^{\mu} \delta^{3}(\vec{r}) .
$$


To check that these are satisfied by Eq. (3.14), first multiply by the matrix $\Gamma$ to obtain

$$
\begin{aligned}
\left(S_{>}^{\mu \nu}(x)-S_{<}^{\mu \nu}(x)\right) \Gamma_{\nu \lambda \rho} & =\left(\delta_{\lambda}^{\mu} \delta_{\rho}^{\kappa}-\delta_{\rho}^{\mu} \delta_{\lambda}^{\kappa}+\eta_{\lambda \rho} \eta^{\mu \kappa}\right. \\
& \left.-i \gamma_{5} \epsilon_{\lambda \rho}{ }^{\mu \kappa}\right) \partial_{\kappa}\left(\sigma_{>}(x)-\sigma_{<}(x)\right) .
\end{aligned}
$$

For canonical momenta, set $\rho=0$ and the time $t=0$. Because $\sigma_{>}(0, \vec{r})=\sigma_{<}(0, \vec{r})$ only the time derivatives on the right (i.e. $\kappa=0$ ) are nonvanishing. This reduces to

$$
\left[\left(S_{>}^{\mu \nu}(x)-S_{<}^{\mu \nu}(x)\right) \Gamma_{\nu \lambda 0}\right]_{t=0}=\delta_{\lambda}^{\mu}\left[\dot{\sigma}_{>}(x)-\dot{\sigma}_{<}(x)\right]_{t=0} .
$$

Thus Eq. (3.18) is satisfied because of the properties of the functions $\sigma_{>}(x)$ and $\sigma_{<}(x)$ from Sec IIIA.

General Covariant Gauge: The results for the gravitino have been displayed in a particular gauge. As was done for the graviton in Sec IIC, one can add a more general gauge-fixing term than used above. The Wightman functions can still be expressed in terms of two derivatives of the functions $\sigma_{>}(x)$ and $\sigma_{<}(x)$ in Eq. (3.8).

\section{DISCUSSION}

It is rather surprising that the large distance effects of massless particles are, in some sense, simpler at $T>0$ than in vacuum. At zero temperature the Wightman function for spinless bosons is

$$
\left.D_{>}(x)\right|_{T=0}=\frac{i}{4 \pi^{2}} \frac{1}{(t-i \epsilon)^{2}-r^{2}},
$$

and for spin $1 / 2$ fermions is $S_{>}(x)=i \gamma^{\mu} \partial_{\mu} \sigma_{>}(x)$, where

$$
\left.\sigma_{>}(x)\right|_{T=0}=\frac{i}{4 \pi^{2}} \frac{1}{(t-i \epsilon)^{2}-r^{2}} .
$$

In perturbative calculations, the slow fall-off at large distances produces long range correlations both in the deep space-like and the deep time-like directions. At $T>0$ the situation is very different as indicated below.

Deep space-like region: For $r-|t| \gg 1 /(\pi T)$ the asymptotic behavior of Eqs. (2.10) is

$$
D_{>}(x) \rightarrow \frac{-i T}{4 \pi r}-\frac{i T}{2 \pi r} e^{-2 \pi T r} \cosh (2 \pi T t)+\ldots
$$

This behavior can also be understood from the Matsubara formalism, 20 [- which has discrete frequencies $\omega_{n}=2 n \pi T$. The static, $n=0$ mode is responsible for the leading $T / r$ behavior. Each higher mode produces an $\exp \left(-\omega_{n} r\right)$ fall-off. For fermions the asymptotic behavior of Eq. (3.8) is

$$
\sigma_{>}(x) \rightarrow \frac{-i T}{2 \pi r} e^{-\pi T r} \cosh (\pi T t)+\ldots
$$

Since fermion have odd frequencies, $\omega_{n}=(2 n+1) \pi T$, the exponential fall-off also comes from $\exp \left(-\omega_{n} r\right)$.
Deep time-like region: In the deep time-like region defined by $|t|-r \gg 1 /(\pi T)$, the asymptotic behaviors of Eqns. (2.10) and (3.8) are

$$
\begin{gathered}
D_{>}(x) \rightarrow \frac{-i T}{2 \pi r} e^{-\pi T|t|} \sinh (2 \pi T r)+\ldots \\
\sigma_{>}(x) \rightarrow \frac{i T}{2 \pi r} e^{-\pi T|t|} \sinh (2 \pi T r)+\ldots
\end{gathered}
$$

It is not obvious why both Wightman functions should fall exponentially in the time-like region and with the same exponent.

All the Wightman functions for higher spin are simply related (by derivatives) to these basic functions. It thus appears that in comparison with zero temperature, the quantitatively large effects of massless particles at nonzero temperature comes predominantly from the $T / r$ behavior of bosons at large spatial separation and from the light cone $t^{2}=r^{2}$. Subsequent publications will explore the physical consequences of these results.

\section{ACKNOWLEDGMENTS}

This work was supported in part by the U.S. National Science Foundation under grant PHY-9900609.

[1] D.Z. Freedman, K. Johnson, and J.I. Latorre, Nucl. Phys. B371, 353 (1992); D.Z. Freedman, G. Grignani, K. Johnson, and N. Ruis, Ann. Phys.218, 75 (1992); D.Z. Freedman, K. Johnson, R. Munoz-Tapia, and X. VilasisCardona, Nucl. Phys. B395, 454 (1993).

[2] N.P. Landsman and Ch. G. van Weert, Phys. Rep. 145, 141 (1987).

[3] M. LeBellac, Thermal Field Theory (Cambridge University Press, Cambridge, England, 1996).

[4] A. Das, Finite Temperature Field Theory (World Scientific, Singapore, 1997).

[5] H.A. Weldon, Phys. Rev. D 62 (2000) hep-ph/0007072.

[6] R. Kubo, J. Phys. Soc. Japan 12, 570 (1957); P. Martin and J. Schwinger, Phys. Rev. 115, 1342 (1959).

[7] F.T. Brandt and J. Frenkel, Phys. Rev. D 58, 085012 (1998); D 47, 4688 (1993).

[8] D.M. Capper, G. Leibbrandt, and M. Ramón Medrano, Phys. Rev. D 8, 4320 (1973).

[9] J. Schwinger, Phys. Rev. 82, 664 (1951).

[10] B.S. DeWitt, Dynamical Theory of Groups and Fields (Gordon and Breach, New York, 1965). 